patients receiving palliative care with support and information in their own home.

- Helping Hands - a non-clinical volunteer support/befriending service supporting patients and their families in the last year of life at home

- Collaborative working with care homes in the NHS, Private and Independent sector to improve end-of-life care

- Working with community services currently being redesigned in several rural areas of Highland to support and improve end of life care closer to home.

This poster presentation will report on all these projects in action with updated results at time of publication.

\section{P-94 IMPROVING ACCESS TO SPECIALIST PALLIATIVE CARE FOR PATIENTS WITH CHRONIC OBSTRUCTIVE PULMONARY DISEASE}

${ }^{1}$ Hazel Coop, ${ }^{2}$ Jan McLachlan, ${ }^{1}$ Helen Ward, ${ }^{2}$ Fran Hakkak. ${ }^{1}$ Royal Wolverhampton NHS Trust, Wolverhampton, UK; ${ }^{2}$ Compton Hospice, Wolverhampton

\subsection{6/bmjspcare-2016-001245.117}

Background Chronic Obstructive Pulmonary Disease (COPD) is a life-limiting condition. In 2012 it contributed to $5.3 \%$ of the total deaths in the United Kingdom. Historically, patients with COPD have not accessed specialist palliative care services. However, there is evidence that patients with COPD have as disabling symptoms as those with cancer. It has been suggested that services need to engage with the COPD population differently due to the insidious disease course and trajectory.

Aim To investigate whether collaborative working has increased access for patients with COPD to palliative care services.

Methods Using the local hospice's CrossCare system we have investigated activity data for Wolverhampton patients in the hospice's different settings; community, day hospice, outpatient and inpatient unit. Data was collected from 2012-13 and 2015-16 to see the impact of a three-pronged strategy of education of the whole health economy, set up of a joint respiratory clinic and a multidisciplinary team meeting to discuss potential palliative patients. Patients with a primary referral diagnosis of COPD were included.

Results Collaborative working has increased access of patients with COPD to all hospice services from 2012/13 to 2015/16. There has been a $283 \%$ increase in patient numbers seen in day hospice. There was an $88 \%$ increase in patients seen by the community team and a $403 \%$ increase in the number of visits. There has been a $286 \%$ increase in patients seen in outpatients by the multidisciplinary team. There were no patients admitted to the inpatient unit in 2012-13 and 13 patients were admitted 201516 with 20 inpatient spells. This enabled four people with COPD to die at the hospice.

Conclusion Patients with COPD in the Wolverhampton area have demonstrable palliative care needs. This is an example of how collaborative working can improve access for this patient group.

\section{P-95 IDENTIFICATION THROUGH INTEGRATION - INCREASING ACCESS TO PALLIATIVE AND END OF LIFE CARE}

Carol Scholes, Louise Saville-King, Mary-Ann Gregory. Hertfordshire Community NHS Trust, Welwyn Garden City, UK

10.1136/bmjspcare-2016-001245.118
Introduction Recognition that someone is entering the last year of life enables access to Palliative and End of Life Care (PEoLC). There is currently under-identification of people in the last year of life.

Aim To increase access to PEoLC through improved identification of the patients known to the Community Trust who are likely to be in the last year of life.

Method In 2014 all Community Trust specialist palliative care nurses (SPCNs) were integrated into seven community integrated care teams, each covering a population of c.100,000 people. The SPCNs were co-located and managed within those separate teams. Targeted PEoLC training was delivered to all staff.

The SPCNs were supported to improve PEoLC within their locality through an internal clinical network which included:

- Weekly Specialist Palliative Care multidisciplinary team meetings

- Senior SPCN leadership and support

- SPCN group clinical supervision

- Specialist Palliative Care clinical governance structure.

Results Two of the seven localities have been the most successful in improving identification of people in the last year of life with a $57 \%$ increase in number.

Abstract P-95 Table 1 Number of people identified as in last year of life on system1 two localities (annual snapshot march)

\begin{tabular}{llll}
\hline Year & 2014 & 2015 & 2016 \\
\hline Number identified & 183 & 223 & 288 \\
\hline
\end{tabular}

These reflections from locality manager and SPCN appear key to improvement:

- Leadership from locality manager on integrating SPCNs

- Weekly locality multidisciplinary clinical meetings to share ideas, opinions, reflect, debrief, give informal education

- Increased visibility improves working relationships - more conversations, trust, respect, team-work, better understanding of roles/workload

- More shared care and joint visits undertaken

- SPCN facilitation of team action learning and clinical supervision

- Offer of uniform to SPCN.

Conclusion Integration of community SPCNs with leadership and support can significantly increase access to PEoLC through improved identification of people in the last year of life.

Next steps A comprehensive education programme is supporting staff development with the aim of further improving access to PEoLC and clinical outcomes. A PEoLC dashboard is in development to provide each locality with required clinical data.

\section{P-96 A SNAPSHOT OF SOCIAL DEPRIVATION AND MULTI- MORBIDITY IN A HOSPICE INPATIENT POPULATION}

${ }^{1}$ Abigail Neal, ${ }^{2}$ Maire O'Riordan, ${ }^{2}$ Julie Spenceley, ${ }^{2}$ Paul Hoy, ${ }^{2}$ Emma Carduff. ${ }^{2}$ University of Glasgow, Glasgow, UK; ${ }^{2}$ Marie Curie Hospice, Glasgow

10.1136/bmjspcare-2016-001245.119

Introduction Recent research has suggested that people living with long-term illnesses in socially deprived areas have poor 
access to specialist palliative care services and poor overall quality of care in the last three months of life.

Aim To describe the inpatient population of a hospice ward and to explore the relationship between level of deprivation and the prevalence of co-morbidities.

Methods Data were collected from patient notes, nursing documentation and computer systems for 30 patients (total number of beds) over 1 week. Scottish Index of Multiple Deprivation quintiles (SIMD 1 - most deprived; SIMD 5 - least deprived) were assigned to assess level of deprivation based on patient postcode. Data were analysed using t-test on Graphpad PRISM.

Findings The sample comprised of 15 female and 15 male patients ranging in age from 56-88 years. Eighteen had been admitted from home, 11 from hospital and one from the hospice outpatient clinic. Primary disease was more commonly malignant than non-malignant (26:4 respectively). Fourteen were from SIMD quintile 1 (most deprived), but only two were from SIMD 5 (least deprived). Patients from areas of high deprivation had nearly double the number of admissions to hospital in the preceding 12 months (SIMD 1-2 mean 3.7 (95\% CI: 2.4-5.1); SIMD 3 and above 2.1 (1.4-2.7) $\mathrm{P}=0.02$ ). The number of co-morbidities ranged from a single disease to nine; there was no correlation with SIMD quintile.

Conclusion The patients captured in this study were predominately from more deprived areas, which reflects the local population. Importantly, these patients had significantly more admissions to hospital compared with patients from less deprived areas. More research is required to understand the reasons for this, including the extent to which these admissions are occurring out of hours, and to determine the level of need for patients and families living and dying in deprived areas.

\section{P-97 HOSPICE WORKING IN PARTNERSHIP WITH THE HOMELESS COMMUNITY}

Sue Scully. St Barnabas Hospice Trust, Lincoln, UK

\subsection{6/bmjspcare-2016-001245.120}

Hospice day therapy staff have been engaging with the homeless community via local services who support them, enabling those who have a life limiting condition(s) the equal opportunity to take control of their lives through the support of our specialist palliative rehabilitation service regardless of their homeless status.

Our aim is to optimise a person's physical function and emotional wellbeing, consistent with their choice, goals and priorities. We work together with patients and those who are important to them using our expertise, to equip them with new tools to maximise wellbeing.

The majority of patients that we care for who are part of this vulnerable and marginalised community, usually present with underlying mental health illnesses and may have alcohol and/or drug addictions. They experience significant ill health and the average age of death is 47 years.

It can be difficult for these people to engage with our services, due to their transient life styles and their psychological and emotional problems. The holistic assessment can be completed at point of contact or at the Day Therapy Centre. If patients do not attend with consent we continue to have contact with their support worker, housing officer.

Other members of staff and students are invited to attend from the Trust. This highlights to other professionals the ongoing difficulties that these patients have and proves that as a Hospice
Trust we are committed to making our service accessible, demonstrating the diverse groups that we support.

With monthly engagement with the services we have forged strong professional relationships with support workers/housing officers and managers and referrals are starting to increase.

Statistics Mean = May 2015-May 2016 monthly contact/discussion with 10 service users

Service users who accessed the centre during this period $=5$

Service users who engaged at point of contact $=4$.

\section{P-98 WE HEAR NOW AND THEN: A SECONDARY QUALITATIVE ANALYSIS OF INNER CITY LONDON MINORITIES' EXPERIENCES WITH HOSPICE CARE}

Munikumar Ramasamy Venkatasalu. Universiti Brunei Darussalam, Gadong, Brunei Darussalam

\subsection{6/bmjspcare-2016-001245.121}

Background Hospices undertake diverse social inclusion strategies to enable hospice access for minority population. Yet, utilisation and access to hospice care among British minorities remain scanty.

Objective To explore perspectives of older British South Asians on use of hospices at the end-of-life.

Design A constructivist grounded theory study.

Methods A secondary qualitative data analysis was taken on views of 55 older British South Asian participants, recruited using purposive sampling. Participants were approached through their community leaders and at open meetings of 11 local South Asian community groups of East London, England. Five focus groups and 29 semi-structured interviews were conducted. Thematic analysis was taken.

Results Three themes arrived: misconceptions, expectations and strategies. Participants described misconceptions related to hospice access including payable hospices and image of nursing home care as hospice care. Analysis also shows that lack of experience often created such misconceptions. Expectations include change in physical and emotional surroundings and enabling home care hospice services. Participants reported various ethniccentred inclusion strategies to promote hospice concept among this minority population.

Conclusion Current social inclusion strategies of hospice seems to promote the spread of hospice concept among these older inner city minorities. However, access and utilisation of hospice services remain 'distant' to real usage. Future strategies should focus microlevel social interventions and mass media based interventions to promote hospice usage among this population.

\section{P-99 A CULTURALLY INCLUSIVE, BAME-COMMUNITY-LED SERVICE FOR THOSE REACHING THE END OF LIFE}

Muhammad Abdullah, Caroline Mawer, Rupina Begum, Abu Mumin. Edencare UK, London, UK

\subsection{6/bmjspcare-2016-001245.122}

Background People from BAME communities are known to have specific palliative care needs. Different cultural approaches may have substantial impacts both within families, and on service use. Despite this, services are often provided 'for', rather than 'developed with' Muslims. In response to this, Muslim community 